\title{
The International Conference on Mathematics, Science, Education and Technology
}

\section{(ICOMSET 2015)}

Education, Mathematics, Science and Technology for Human and Natural Resources

October 22, 2015

Inna Muara Hotel and Convention Center Padang, Indonesia

Faculty of Mathematics and Science State University of Padang Padang, Indonesia 


\title{
OPTIMITION OF LEAST SQUARES METHODS SMOOTH CONSTRAIN USING OCCAM'S INVERSION GEOELECTRIC RESISTIVITY DIPOLE-DIPOLE CONSFIGURATION FOR ESTIMATION SLIP SURFACE
}

\author{
Akmam ${ }^{1}$, Irefia, R., D., ${ }^{2}$, Silvia $D^{2}{ }^{2}$, S., Jemmy, $R^{2}$. \\ ${ }^{1}$ Physics Department, Faculty of Mathematics and Science, State University of Padang, Indonesia \\ ${ }^{2}$ Student of Physics Department, Master Program, Faculty of Mathematics and Science, State University of \\ Padang, Indonesia \\ Akmam_db@fmipa.unp.ac.id
}

\begin{abstract}
The volumes of the landslide depend on the depth of the slide surface. The depth of the slide surface can be predicted by using the least squares inversion methods of data geoelectrical. Method of Least Squares Inversion of the data Geoelectrical cannot get smooth results. Based on the above, the aims was optimalitation leastsquares smooth constrain inversion methods with Occam's inversion for geoelectrical data and to determine the depth and tilt angle of the slide surface in in Bukit Lantiak Padang . This exploratory study was use the method Geoelectrical resistivity Dipole-Dipole configuration. The depth and tilt angle of the slide surface in Bukit Lantiak Padang was interpretated by combined Occam's inversion with least-squares smooth constrain inversion methods. The type of slip surface is translation slip with 33.450 tilt angle and 19.3 meters in depth. Research show that the result interpretation by used combined Occam inversion with least-squares smooth constrain inversion methods more sharpness than least-squares smooth constrain inversion.
\end{abstract}

Index Terms: Least- Squares, Smooth-Constrain, Occam’s Inversion, Geoelectric data

\section{INTRODUCTION}

Landslides is a natural phenomenon that is often take place on undulating topography. Landslide caused much loss of life and infrastructure ${ }^{[1]}$, however usually pasca incident community are rebuild damaged infrastructure such as houses. The quantity of damage on landslide are depend on the volume and speed of the landslide. Volume landslide depends on the depth of sliding plane, while the landslide speed depends on the angle of the field of landslide. These problems need attention and scientific study about quantity of next landslide. This study is importent as the initial assessment of landslide disaster mitigation. Boundaries between rock wheathering or soil that was eroded with the impermeable rocks (the hard rock beneath which acts as a foundation) is called slip surface.

In the resistivity methods, slip surface are identified with of two layers of rock that have contrast resistivity ${ }^{[1],[2],[3],[4]}$. Slip surface usually consisting of a hard coating with a layer of soft and groundwater ${ }^{[5]}$. Slip surface usually consisting of rock that is low permeability that weathering rock (soil) is move on it. Structure of rock as the slip surface can be seen from the value of resistivity of rock. Resistivity of rock as the slip surface around (200-100) $\Omega \mathrm{m}^{\text {[6], [7] }}$.

Resistivity contrast between two types of rocks adjacent to the slip surface is influenced by rainfall and slope of hill or mountain ${ }^{[2],[7]}$. Landsilide surface on the hill or mountain that have a slope angle greater than $25^{\circ}$ is divided into two types, namely the rotation slip and the translation slip (slip surface parallel to the surface of the slope ${ }^{[8]}$. The rotation slip surface is the sliding plane that the landslide material a rotation movement above this plane. Translation slip surface is the surface that landslide material moves translational and moves in block form.

The estimation of true resistivity distribution against depth from the apparent resistivity data essentially lead to solving the inverse problem. Further, relation between the observed "apparent resistivity" and the model parameters ("true resistivity" and "layer thickness") is non-linear. Forward mathematical models are generally used to relate the measured data (here apparent resistivity) to desired model parameters (true resistivity and layer thickness). Essentially, the forward modeling is a process of predicting results of measurements on the basis of some general principle or model and specific conditions relevant to the problem. Inverse modeling, in contrast starts with the data and a general principle or a model, in which the model parameters are estimated by minimizing the error set up between the data and model parameters ${ }^{[9]}$. During the past decades researchers have made several attempts to solve the resistivity inverse problems [10],[11],[12]. These algorithms critically depend on the initial parameter chosen for it. These methods are also computationally expensive and sometimes found unfeasible for processing voluminous amount of data. Thus it is imperative to search for solving non-linear resistivity inverse problems.

\section{RESISTIVITY METHODS}

In the resistivity method, current is induced directly into the ground through a pair of current electrodes and resulting voltage difference is measured between a pair of potential electrodes. The 
method provides the apparent resistivity distribution against depth. The depth of penetration of electrical signal is generally found to be approximately onethird of the distance between the electrode separations. The layered structures in and around the region have impacted on the near surface distribution pattern of electrical properties. In this method, current is induced directly into the ground through a pair of current electrodes and resulting voltage difference is measured between a pair of potential electrodes. The method provides the apparent resistivity distribution against depth. The depth of penetration of electrical signal is generally found to be approximately one-fitth of the distance between the electrode separations.

The subsurface geology is usually complex, subtle and multi-scale such that spatial variations can change rapidly both laterally along the survey profiles and vertically with depths. Thus, a closely spaced grid of observation points is required for the accurate characterization, high spatial resolution and good target definition of such highly heterogeneous subsurface. Survey design must take into account the capabilities of the data acquisition system, heterogeneity of the subsurface electrical conductivity and the required resolution. Other factors to be considered are the areal extent of the site to be investigated, the cost of the survey and the time required to complete the survey ${ }^{[13],[14],[15]}$.

If the point electrode delivering current (I amperes) is located at the surface of the homogeneous isotropic medium, all the current now flows through a hemispherical surface in the lower medium because and the air above earth has zero conductivity. The flow of electric current in the earth as Figure $2^{\text {[14],[15]. }}$

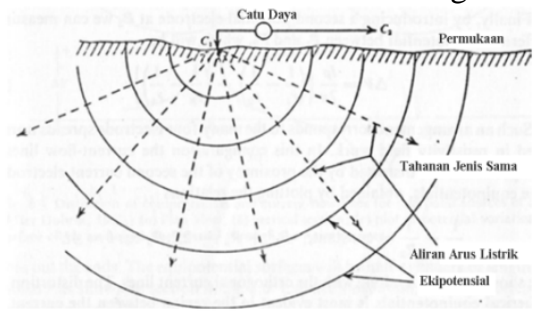

Figure 2. Point source of current at the surface a homogeneous medium

If the current source and potential are located on surface, the horizontal boundary separating two media (e.g., the upper resistivity $\rho_{1}$, the lower $\rho_{2}$ ), the electrical potential $\mathrm{V}(\mathrm{x})$ on the surface $(\mathrm{z}=0)$ of $\mathrm{a}$ layered earth at a distance $\mathrm{s}$ from a grounded electrode carrying a current $\mathrm{I}$, at point distand $\mathrm{x}$ from current electrodes ${ }^{[16],[14],[35]}$ can be write the equation as follows

$$
V(x)=\frac{1}{2 \pi} \int_{0}^{\infty} T_{1}(\lambda) J_{0}(\lambda x) d \lambda
$$

where $\mathrm{J}_{0}(\lambda \mathrm{x})$ is the zeroeth order Bessel function of the first kind, $T_{1}(\lambda)$ is the electrical impedance at the surface that is the resistivity transform function of layer resistivities and thicknesses ${ }^{[17]}$, and $\lambda$ denotes the integral variable ${ }^{[18]}$ (specifically for dipole-dipole configuration is half of the positive current electrode spacing of the electrode potential ${ }^{[19]}$ ). The resistivity transform function to extract the resistivity kernel function $K_{1}(\lambda)$ representing the deviations in response of the layered earth from that of a homogeneous halfspace.

Irregularities on the response of the layered earth homogeneous half-space can be represented by the function of the resistivity transformation into Kernel function $K_{1}(\lambda)$ of resistivity ${ }^{[17]}$. Recurrence relationship of the resistivity transform function impendasi electrical resistivity $\mathrm{T}(\lambda)$ can be written as follow

$$
T_{i}(\lambda)=\frac{T_{i+1}(\lambda)+\rho_{i} \tanh \left(\lambda_{i} h_{i}\right)}{\left[1+T_{i+1}(\lambda) \tanh \left(\lambda h_{i} / \rho_{i}\right]\right.}, i=n-1 \ldots 1
$$

while $n$ denotes the number of layers, $\rho$ i denotes the true resistivity and thickness of the i-th layer, respectively. Electrical impedance at the earth surface (the air-earth interface) can be formulated by

$$
T_{1}(\lambda)=\rho_{1}\left[1+2 K_{1}(\lambda)\right] \text {, }
$$

while electrical impedance at the top of the ith layer [18] can be write as follows

$$
T_{i}(\lambda)=\rho_{i}\left[1+2 K_{i}(\lambda)\right]
$$

Earth is not homogenous in resistivity, so the relationship between the apparent resistivity ( $\rho$ and the layer parameters (e.g. layer thickness, layer true resistivity) can be expressed by an integral equation considering an earth model consisting of homogeneous and isotropic layers can be write the equation as follows ${ }^{[12],[37]}$.

$$
\rho_{a}(x)=x^{2} \int_{0}^{\infty} T(\lambda) J_{1}(\lambda x) \lambda d \lambda
$$

where $\rho$ a denotes an apparent resistivity.

To Calculate Equation (5) can be under taken with a linear filter method is generally expressed as following equation.

$$
\rho_{a}(x)=\sum_{k} T_{k}(\lambda) f_{k}
$$

while fk denotes a linear filter coefficient derived by Ghosh ${ }^{[20]}$. Equation (6) shows the relationship between the apparent resistivity $\left(\rho_{a}\right)$ with the parameters of the model resistivity and thickness of the layer $\left(\rho_{k}, h_{k}\right)$ is very linear [21], so to estimate the apparent resistivity to true resistivity inversion required non-linear solution. Next, base on the equation (6) can be obtained the simple expression apparent resistivity that measured by symmetrical dipole-dipole configurations in discrete Green's function as follows ${ }^{[18]}$

$$
\rho_{a}(x)=\sum_{j=0}^{\infty} T_{1 j}\left(\frac{m}{m-1} \frac{x}{\sqrt{\left(\varepsilon_{j}^{2}+x^{2}\right)}}-\frac{1}{m-1} \frac{m x}{\sqrt{\left(\varepsilon_{j}^{2}+(m x)^{2}\right)}}\right)
$$

which is the Green's function of a point source, $\varepsilon_{\mathrm{j}}$ being a real constant and $\mathrm{m}$ denotes specific electrode configurations. 


\section{OCCAM'S INVERSION IN LEAST-SQUARE SMOOTH CONSTRAIN}

The behavior of the electrical properties of the earth's subsurface (resistivity and thickness) were studied based on the electrical parameters of the structure below the earth's surface ${ }^{22]}$. Layer of earth is modeled in the form of a series of horizontal layers with constant resistivity. The real resistivity distribution estimation (true resistivity) to a depth based on the apparent resistivity data (apparent resistivity) can be solved by mathematical and statistical techniques to obtain information about the physical properties of the subsurface of the earth ${ }^{[23]}$. Inversion method Least-Square 2-D can be use to interpret of the subsurface resistivity structure ${ }^{[24,[25]}$, but the results of inversion have not shown results in a smooth response ${ }^{[13]}$.

The earth is not a homogeneous medium due to subsurface resistivity is changes continuously with depth. The local layer parameters characterizing the model are frequently estimated by using apparent resistivity data (sounding curves) determined at relatively sparse stations. In order to ensure the fulfillment of a 1-D assumption as much as possible, the measurement electrode arrays are deployed in the strike direction of the geological structure ${ }^{[19],[20]}$. By the method all observation data (measured with an electrode array) from each VES station along the survey profile are integrated into one inversion procedure ${ }^{[36]}$. The lateral change of the model parameters (the dependence of layer resistivities and thicknesses on the lateral coordinates) are approximated by series expansion. The main problem in the inversion approach is the result of inversion does not produce a unique solution ${ }^{[9],[22]}$. Such inversion displays the same response from three or more different models. This can lead to errors in the interpretation of the parameters. In order to give better approximation for geological models with slow lateral variations a new inversion algorithm, such as Robust inversion, Occam's inversion and joint inversion [9],[26]

An alternative approach can be used to parameterize the amount of geoelectric earth layers horizontally with a continuous resistivity profiles around sounding using electromagnetic data which is linear [9],[11],[27]. The relationship between the observation data (apparent resistivity) and model parameters (true resistivity and layer thickness) is not linear ${ }^{[37]}$. Standard linear inverse approach to solving the problems of non-linear inversion in Geophysics in general are developed based on the iteration process. In inversion process, the number of iterations and error interpretation results generally depend on the value of the initial guess ${ }^{[11],[27]}$.

The Levenberg-Marquardt algorithm adaptively varies the parameter updates between the gradient descent update and the Gauss-Newton update ${ }^{[28]}$.

$$
\left[\mathrm{J}^{\mathrm{T}} \mathrm{WJ}+\lambda \mathrm{I}\right] \Delta m_{k}=\mathrm{J}^{\mathrm{T}} \mathrm{Wd}
$$

where $I$ is the identity matrix and $\mu$ is Marquardt factor or damping factor. The parameter $\lambda$ is initialized to be large. If an iteration happens to result in a worse approximation, $\lambda$ is increased. As the solution approaches the minimum, $\lambda$ is decreased, the Levenberg-Marquardt method approaches the GaussNewton method, and the solution typically converges rapidly to the local minimum ${ }^{[23] \text {. }}$

If data measured in geoelectrical modeling the Marquardt inversion was expressed as $d=$ [ such as $\mathrm{s}$ apparent resistivity with $\mathrm{i}=1,2, \ldots, \mathrm{n}$ and $\mathrm{n}$ is the number of data. Model resistivity and thickness of the subsurface is $\Delta \mathrm{m}_{\mathrm{k}}=\left[\rho_{\mathrm{k}}, \mathrm{h}_{\mathrm{k}}\right]$, while $\mathrm{k}=1,2, \ldots, \mathrm{n}$ and $\mathrm{n}$ is the number of layers. Marquardt inversion method of applying iterative methods as well as the Gauss-Newton method, the difference lies in the addition of damping factor $\mu$ scalar multiplication with the identity matrix $\mathrm{I}^{[29],[30]}$. The term damping or attenuation to estimate model parameters as stated in Equation (8) relates to dampen the instability that might arise due to limited data on inversion including underdetermined $^{[31]}$.

To obtain a unique solution inversion various constraints must be added. To reduce the nonuniqueness problem and to achieve a faster computer code, several authors have combined the use of 2-D and 1-D calculations serving 1-D grid laterally with inversion procedure requiring too much numbers iterations ${ }^{[27]}$. Numbers iterations process was reduced by modifying the Occam's inversion with cutting singuler value, however this inversion method can not find out a conductive thin layer and a conductive layer which was covered by resistive layers ${ }^{[32]}$. This information indicates that this method can not use to investigated landslide. If this method will use to data interpretation, it is necessary to improve. Another problem is the result of inversion of resistivity sounding a little change in the value of the model parameters lead to large changes in the results of inversion ${ }^{[12]}$. This condition will be overcome by using Occam inversion that is inserted into the equation linear least-squares inversion as a regulator of the process of calculating a damping factor. Damping factor for the linear least-squares inversion damped ${ }^{[30]}$ as follows

$$
\mathrm{m}=\left[\mathrm{G}^{\mathrm{T}} \mathrm{G}+\varepsilon^{2} \mathrm{~W}\right]^{-1} \mathrm{G}^{\mathrm{T}} \mathrm{d}
$$

where $m$ is the vector correction parameter, $d$ is the vector difference data $G$ Jacobian matrix containing the partial diffrensial data to the model parameters and $\varepsilon$ is called the damping factor. The damping factor is used to control the convergence and the obtained solution ${ }^{[16]}$. In cases of resistivity data inversion, partial differential price is expected to model parameters are not too large so that the price difference inversion model parameters can be minimized. $\mathrm{W}_{\mathrm{m}}$ matrix is a square matrix $(\mathrm{M} \times \mathrm{M})$ with the same size as the model

$$
\underline{W}=\operatorname{diag}\left\{\rho_{1}, \rho_{2}, \ldots \ldots \ldots \rho_{M}\right\}
$$

Minimization of errors on a regular linear inversion expanded to minimization of the prediction 
error data and error solutions. If the damping factor used is very small or close to zero, the prediction error will be minimized [20],[29]. That is, the addition of damping factor in the inversion process can stabilize the inversion process on the matrix inversion ${ }^{[16]}$.

Non-uniqueness problem inversion results are minimized by using the minimum norm as constrain models and minimize the objective function related to geological conditions desired. Results inversion models will thus provide information in accordance with the desired geological conditions. Constraints in this case are anomalous resistivity on the slick field using electrode configuration dipole-dipole. Parameters of model modified iteratively by using the equation as follow

$$
\Delta m^{k}=\underline{W}_{k}^{-1} \underline{G}^{T}\left[\underline{G} \underline{W}_{k}^{-1} \underline{G}^{T}+\lambda \underline{I}\right]^{-1}\left(x-\underline{G} m^{k}\right)
$$

For a 2-D model the earth is parameterized by means of a grid of rectangular prisms, each having a uniform conductivity ${ }^{[27]}$. The grid is terminated laterally by uniform layers and below by prisms elongated with depth. This grid is referred to as the regularization mesh. The individual blocks are made smaller than the data resolution length so that the locations of the block boundaries do not affect the final model ${ }^{[33]}$. To perform the forward calculations required by the inversion scheme a finite-element ${ }^{[30]}$.

However, the regularization mesh contains a much larger number of conductivity regions than is normally used for 2-D models based on assumed geologic structures, and so using several nodes for each conductivity element would be computationally expensive. The smooth inversion scheme will prevent large conductivity contrasts from appearing in the model, and several inversions have been conducted using no more nodes than defined by the regularization mesh. However, it is usually desirable to insert more nodes within the regularization mesh to maintain accuracy in the forward code, especially at the edges of the grid [19]. To suppress model structure not required by the data, the model roughness must be minimized. For a 2-D structure with $\mathrm{x}$ in the direction of the strike axis a measure of the model roughness may be given by

$$
R_{1}=\left\|\partial_{y} m\right\|^{2}+\left\|\partial_{z}\right\|^{2}
$$

where $\mathrm{m}$ is the vector of model parameters, $\partial \mathrm{y}$ is a roughening matrix which differences the model parameters of laterally adjacent prisms, and $\partial \mathrm{z}$ is a roughening matrix which differences the model parameters of vertically adjacent prisms ${ }^{[19],[33],[34],[35]}$. This is the expression for a first derivative roughness penalty. The penalty for the second derivative roughness is given by

$$
R_{2}=\left\|\partial_{y}^{2} m\right\|^{2}+\left\|\partial_{z}^{2} m\right\|^{2}
$$

Since the model grid is terminated by uniform layers at the sides and uniform blocks below, first derivative smoothing best matches the boundary conditions imposed by the forward code. Therefore only the $\mathrm{R}_{1}$, roughness penalty will be discussed. The vertical scale of the prisms is exponentially increased as a function of depth in order to coincide with the loss of resolving power, so that this is equivalent to increasing the penalty for roughness as a function of depth. The horizontal block boundaries and node spacing in the forward code extend to depth and are constrained by the requirement of having a fine mesh near the surface. Since the block widths remain constant, the horizontal damping factors in the roughening matrix are adjusted to ensure that resolved features are not elongated in the vertical direction. That is, as the vertical scale of the prisms increases, a greater penalty must be given to differences in model parameters between laterally adjacent blocks [19], [33], [35]. In order to quickly reach the converging and inversion results reflect the expected geological conditions Equation (13) can be modified into $\Delta m_{i+1}^{k}=\left\lfloor\mu\left(\underline{\partial}_{y}^{T} \underline{\partial}_{y}+\underline{\partial}_{z}^{T} \underline{\partial}_{z}\right)+\underline{W}_{k}^{-1} \underline{G}^{T}\left(\underline{G} \underline{W}_{k}^{-1} \underline{G}^{T}+\lambda \underline{I}\right)^{-1}\left(x-\underline{G} m_{i}^{k}\right)\right\rfloor$ (14)

An unvaried search is conducted along model on each iteration in order to find a model that minimizes the misfit to the data until the desired ttolerance is obtained.

\section{METHODS}

Model inversion obtained verified by resistivity data measurement in Lantiak Bukit Padang. Data was collected using a multi-electrode system resistymeter (ARES). The use of multi-electrode systems for data acquisition in Geoelectric resistivity surveys has led to a dramatic increase in field productivity as well as increased quality and reliability of subsurface resistivity information obtained. Multi-electrode systems were used computer-controlled multielectrode systems with automatic measurements and data quality control, which has tremendous impact on the quality of the data and the speed with which they are collected. Multi-channel transmitter and receiver systems are now being used in simultaneously carrying out series of measurements. This research has three parameters, which was parameter which was measured in survey (electric current, electric potential, and spaced electrodes), calculated parameter I which was the apparent resistivity and interpretation parameter (true resistivity and the thickness of the subsurface).

This study was divided into three stages first, do the preparation phase assessment literature on theories that support. Survey is under taken to determine the trajectory measurements and determine the geological measurement area. Second, the planning phase is to design the measurement to be performed. Third, the implementation phase is to do measurements or data acquisition measurements in accordance with the design that has made as many as three tracks. Measurements carried out by Dipole-dipole configuration, because this configuration has a current penetration is deep enough that $1 / 5$ of current electrode spacing that used and sensitive to vertical 
resolution. The arrangement of the electrodes on Dipole-dipole configuration shown in Figure 1

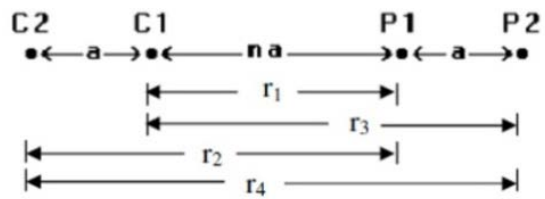

Figure 1. Structure of Dipole-dipole electrode configuration.

Apparent resistivity measurement results calculated

$$
\rho_{a}=\pi n(n+1)(n+2) a \frac{\Delta V}{I}
$$

Where a denotes distance between the two nearest electrodes, $n$ denotes integer number (e.g. $n=1$...6) and na denotes space the positive current electrode and the negative potential electrode. Apparent resistivity was interpreted by the Least-Square inversion method that was optimized with Occam's inversion. Occam's inversion is used to get the value of damping for Least-Squares smooth constraint inversion. Based on 2D cross sections was obtained true resistivity of the earth's subsurface. Based on the true resistivity that obtained, geological structure, and table references were estimated resistivity of rock in Bukitlantiak Padang. Based on 2D cross-sectional, we can estimate type, depth and tilt angle of slip surface. All interpretation of data was under taken by Matlab software version 7.01 and the RES2DINV with Dogle of Sri Gutomo USM Malaysia ${ }^{[38]}$

\section{RESULTS AND DISCUSSION}

To determine whether the inversion method developed to function properly, it has conducted trials use this method in some survey data geoelectric resistivity. First, the method is tested to interpret the data on the measurement of strike $1\left(00^{\circ} 58\right.$ ' $16^{\prime \prime} \mathrm{S}$ and $100^{\circ} 22^{\prime} 8^{\prime \prime} \mathrm{E}$ to $00^{\circ} 58^{\prime} 05.4^{\prime \prime S}$ and $\left.100^{\circ} 22^{\prime} 14.5^{\prime \prime} \mathrm{E}\right)$. This strike has an altitude at (79-125) meters above sea level. The 2D model cross-sectional data on strike 1 is shown in Figure 2.

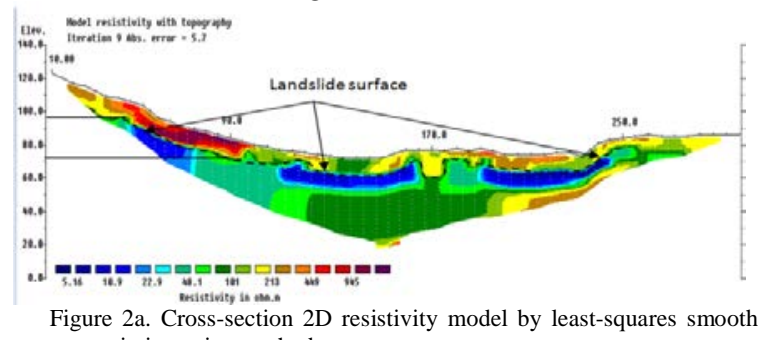
constrain inversion methods

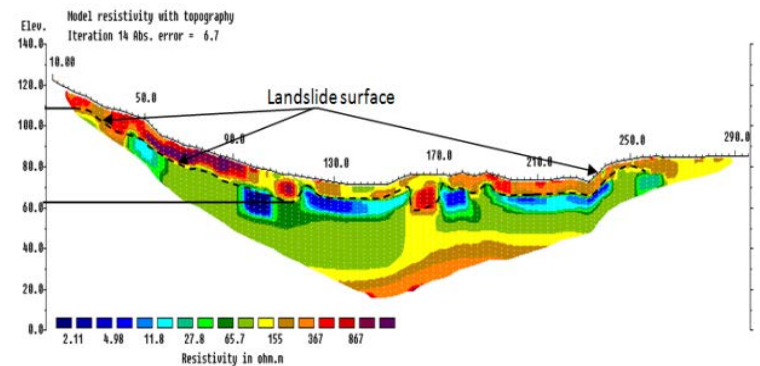

Figure 2b. Cross-section 2D resistivity model by combined Occam's inversion and least-squares smooth constrain inversion methods
Cross-section 2D resistivity model show that structural model of 2-D resistivity was interrelated by Occam's inversion method that combined with smooth constrained least-squares inversion (Figure 2.b) more sharpness than structural model of 2-D resistivity was interrelated by Least-squares constrain smooth inversion methods (Figure 2.a). The tilt angle obtained by Occam's inversion method that combined with smooth constrained least-squares inversion (Figure 2.b) greater than tilt angle obtained by Least-squares constrain smooth inversion methods (Figure 2.a). Resistivity at strike 1 is around 1.21 ohm.m to $2005.76 \mathrm{ohm}$.m. Cross-section that is green in color (resistivity is $19.52 \mathrm{ohm} . \mathrm{m}$ to $27.81 \mathrm{ohm} . \mathrm{m}$ ) was estimated as clay. Thin layer which greener color (a dashed black line) is estimated as slip surface. Slip surface on the strike has an altitude (78-100) meters above sea level and the tilt angle is 33.980. Type of slip surface on strike 1 is translation slip.

Next, this method was used to interpret data on strike $2\left(00^{0} 58^{\prime} 16^{\prime \prime S}\right.$ and $100^{0} 22^{\prime} 8^{\prime \prime E}$ to $00^{0} 58^{\prime} 05.4^{\prime \prime} \mathrm{S}$ and $\left.100^{\circ} 22^{\prime} 14.5^{\prime \prime E}\right)$. This strike has an altitude 71 115 meters above sea level. The 2D model crosssectional data on strike 2 is shown in Figure 3.

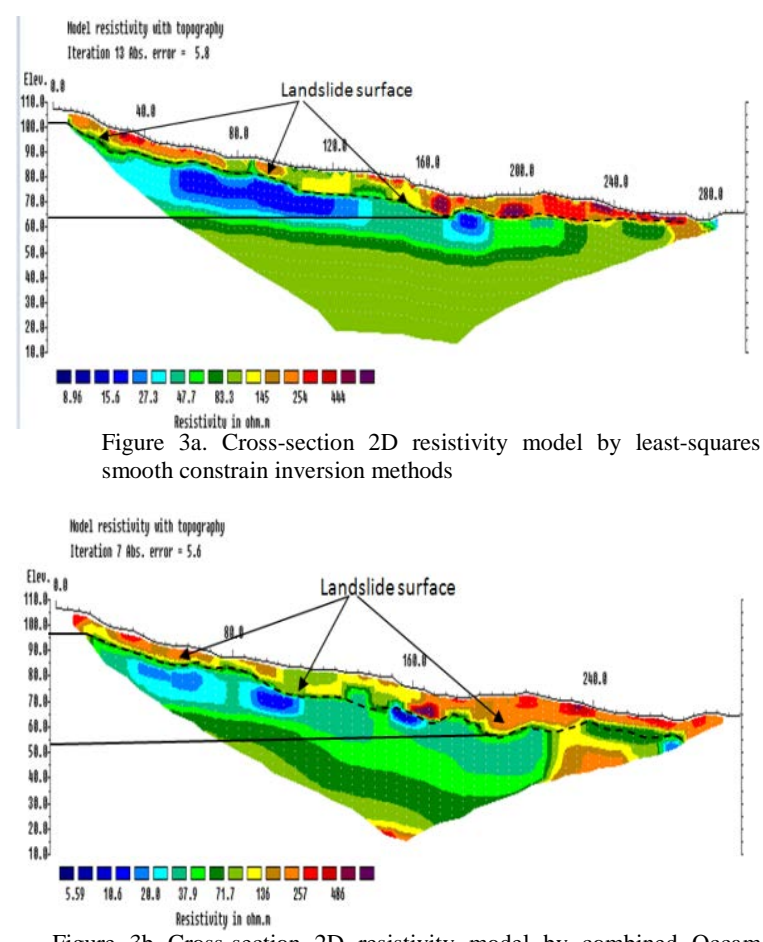

Figure 3b Cross-section 2D resistivity model by combined Occam inversion with least-squares smooth constrain inversion methods

Cross-section 2D resistivity model show that structural model of 2-D resistivity was interrelated by Occam's inversion method that combined with smooth constrained least-squares inversion (Figure 3.b) more sharpness than structural model of 2-D resistivity was interrelated by Least-squares constrain smooth inversion methods (Figure 3.a). The tilt angle obtained by Occam's inversion method that combined with smooth constrained least-squares inversion (Figure 3.b) greater than tilt angle obtained 
by Least-squares constrain smooth inversion methods (Figure 3.a). Resistivity of strike 2 around (1.43 Ohm.m - 1879.54 Ohm.m). Cross-section that is green in color (resistivity is 25.5 ohm.m - 37.9 ohm.m) was estimated as clay. Thin layer which greener color (dashed black line) is estimated as slip surface. Slip surface on the strike 2 has an altitude 62 - 106 meters above sea level and the tilt angle is 32.680. Type of slip surface on strike 2 is translation slip. The percentage of errors interpretation by Occam's inversion method that combined with smooth constrained least-squares inversion on 14th iteration is around $5.6 \%-6.7 \%$.

The overall study results showed that the crosssection 2D resistivity model show that structural model of 2-D resistivity was interrelated by Occam's inversion method that combined with smooth constrained least-squares inversion more sharpness than structural model of 2-D resistivity was interrelated by Least-squares constrain smooth inversion methods. Results obtained by using methods developed sharper than the results obtained by using the least-squares inversion smooth constrains methods which has been widely used ${ }^{[35]}$. Type of sliding plane obtained is the type of translation. It is located above the sliding plane parallel to the hard rock slope. The characteristics are geoelectric sliding plane is the presence of a layer that has a value large enough resistivity between two adjacent layers. Layer with low resistivity flanked by two layers which have a high resistivity. Usually the rock that serves as a sliding plane is the clay because the clay absorbs water during the rainy season but the slow passing of water so that the load of rocks increases. Water that accumulates on clay which is above the hard rock lead becomes hard rock slippery. Slippery clay formed acts as a field of experience weathering material above it moves. Material moving on a slippery field is called as the landslide material. The boundary between materials that is stationary with a moving material called sliding plane. Therefore the need for anticipatory action to prevent landslides

\section{CONCLUSION}

Study results showed that the cross-section 2D resistivity model show that structural model of 2-D resistivity was interpretive by Occam's inversion method that combined with smooth constrained leastsquares inversion more sharpness than structural model of 2-D resistivity was interpretive by Leastsquares constrain smooth inversion methods. Type of sliding plane obtained is the type of translation. The percentage of errors interpretation by Occam's inversion method that combined with smooth constrained least-squares inversion on 14th iteration is around $5.6 \%-6.7 \%$.

\section{ACKNOWLEDGEMENTS}

My thanks go to students from the Department of Physics, Faculty of Mathematics and Sciences Padang State University who helped carry out the survey with the resistivity method in Bukitlantik. Survey data collection was funded by the Directorate General of Higher Education through Student Creativity Week that guided the first author. Thanks also to the Ministry of Energy and Mineral Resources of West Sumatra who allowed us to use the land to carry out a survey.

\section{REFERENCES}

[1]. Chi-Yuen Wang, Sundaram, P.N., Goodman, R.E., Electrical Resistivity Charges in Rock During Frictional Sliding, Rock Friction and Earthquakes Prediction Contribution to Cuurent Research in Geophysics, 1978, 6, 717-731

[2]. Malcom J. Joab, and Martin Andrews, Investigating Slope Failures Using Electrical Resistivity: Case Studies. The Journal of the Association of Professional Engineers of Trinidad and Tobago, 2009, 38(1),66-75

[3] Chambers, J.E., P.B. Wilkinson, D.A. Gunn, R.D. Ogilvy, G.S. Ghataora, M.P.N. Burow,Tilden, S.R, Non-invasive characterization and monitoring of earth embankments using Electrical Resistivity Tomography (ERT)., British Geological Survey, 2007.e-Journal, download September 2014.

[4]. Kuldeep Pareta, Upasana Pareta, Landslide Modeling and Susceptibility Mapping of Giri River Watershed, Himachal (India), International Jurnal od Science and Technology, 2013, 1(2)

[5]. William Lowrie, Fundamental of Geophysics Second Edition, Cambridge University Press, 2007.

[6]. Fransheri, A., Ludyig, K., and Foto, D. Geophysical landslide investigation And Prediction In The Hydrotechnical Works. Journal Of The Balkan Geophysical Society. 1998. 1(3). Tirana Albania.

[7]. Perrone, A., Sabatino, P., and Vincenzo, L. Electrical Resistivity Tomographies For Landslide Monitoring: a Review. Berichte Geol. B, 2012.-A.93. ISSN 1017-8880

[8]. VSI (Vulcanology Survey of Indonesia). Pengenalan Gerakan Tanah. Departemen Energi dan Sumber Daya Mineral, darihttp:// ESDM.ac.id/ pengenalan_gerakan_tanah/VSI, 2005, (diunduh pada 3 Februari 2014).

[9]. Vozoff, K., Jupp, D.L.B.,. Joint inversion of geophysical data. Geophysical Journal of the 
Royal Astronomical Society 1975, 42, 977991

[10]. Hermance, J. F., Pederson, J., Least-Square Inversion of One-Dimensional Magnetotelluric Data, an Assessment of Procedures Employed by Brown University, Geophysics Journal Astronomi, 1986, 8, 87-196

[11]. Akmam, Doddy Sutarno, Pemodelan Inversi Satu Dimensi Data Magnetotellurik Memodifikasi Inversi Occam dengan Pemotongan Nilai Singuler, Jurnal Kontribusi Fisika Indonesia, Jurusan Fisika FMIPA ITB Bandung, 1997, 8(2), 97-106.

[12]. Maiti,S. G. Gupta, , V. C. Erram, and R. K. Tiwari, Inversion of Schlumberger resistivity sounding data from the critically dynamic Koyna region using the Hybrid Monte Carlobased neural network approach, Non Linear Process Geophysics, 2011, 18, 179-192, www.nonlin-processes-geophys.net

[13]. Gyulai, A, Tamás Ormos, Mihály Dobroka, A Quick 2-D Geoelectric Inversion Method Using Series Expansion, Journal of Applied Geophysics, 2010, xxx,xxx ww.elsevier.com/ locate/jappgeo

[14]. Telford, W.M. Geldart, L.P, Sheriff R.E and Keys, D.A. Applied Geophysics. USA: Cambridge University Press. 1976.

[15]. Reynolds, J.M. An Introduction to Applied and Environmental Geophysics. New York: Jhon Geophysicsin Hidrogeological and Wiley and Sons Ltd, 1997.

[16]. Ekinci, Y. L. and Demirci, A.: A damped leastsquares inversion program for the interpretation of Schlumberger sounding curves, Journal of Applied Sciences, 8(22), 4070-4078, doi:10.3923/jas.2008.4070.4078, 2008.

[17]. Koefoed, O., A fast method for determining the layer distribution from the raised kernel function: Geophys. Prospecting, Volume 18, 564 570. 1970,

[18]. Gupta, S.K, Sri Niwas and Vinod K. Gaur, Straightforward inversion of vertical electrical sounding data, Jurnal Geophysics, 1997, 62(3), 775-785

[19]. Constable, S.C, Robert L. Parker, and Catherine G. Constable, Occam's Inversion: A Practical Algorithm For Generatlng Smooth Models From Electromagnetic Sounding Data, Jurnal Geophysics, 1987, 52(3), 289-300

[20]. Grandis, Hendra, Pengantar Pemodelan Inversi Geofisika, Himpunan Ahli Geofisika Indonesia (HAGI), 2009.
[21]. Maiti, S. and Tiwari, R. K.: A hybrid Monte Carlo method based artificial neural networks approach for rock boundaries identification: $A$ case study from the KTB bore hole, Pure Appl. Geo- phys., 166, 2059-2090, 2009

[22] Macias, C. C., Sen, M. K., and Stoffa, P. L.: Artificial neu- ral networks for parameter estimation in geophysics, Geophys. Prospect., 48, 21-47, 2000.

. [23] Donald, W. Marquardt, An Algorithm for LeastSquares Estimation of Nonlinear Parameters, Journal of the Society for Industrial and Appliied Mathematics, Vol. 11, No. 2, Juni 1963, 431-441.

[24]. Akmam, Studi Terhadap Air Tanah Padang Simawang Kecamatan Rambatan dengan Metoda Inversi Least-Square Dua Dimensi, Jurnal Sainstek, 2006, VIII(2,), 75-84

[25]. Loke, H.M, Res2Dinv Rapid 2D Resistivity \& IP Inversion, for Windows XP/Vista, Goetoma Software Malaysia, 2009

[26]. Møller, I, Bo H. Jacobsen, and Niels B. Christensen, Rapid inversion of 2-D Geoelectrical Data by Multichannel Deconvolution, Geophysics,2014,66(3),800808

[27]. Smith. J. T. and Booker. J. R.. . Magnetotelluric inversion for minimum structure: Geophysics, 1988, 53, 1565-1576.

[28]. Henri P. Gavin, The Levenberg-Marquardt method for nonlinear least squarescurve-fitting problems, Department of Civil and Environmental Engineering Duke UniversityOctober 9, 213. 1-17

[29] Hans Bruun Nielsen, Damping Parameter In Marquardt's Method, Imm Department Of Mathematical Modelling, Technical University of Denmark DK-2800 Lyngby - Denmark, 1999

[30]. Wannamaker. P. E., Stodt, J. A., and Rijo, L., 1987, A stable finite-element solution for two-dimensional magnetotelluric modeling: Geophys. J. Roy. Astr. Sot., 88, 277-296.

[31]. Abdul-Nafiu, A.K, Nawawi, M. M. N., Khiruddin, A, Ishola, K. S and Abdulrahman, A, Effects Of Electrode Spacing And Inversion Techniques On The Efficacy Of $2 d$ Resistivity Imaging To Delineate Subsurface Features, American Journal of Applied Sciences, 2013,10 (1): 64-72,

[32]. Akmam dan Nofi, Y,S., Analisis Struktur Batuan Dengan Metoda Inversi SmoothnessConstrained Least-Squares Data Geolistrik Konfigurasi Schlumberger Di Universitas 
Negeri Padang Kampus Air Tawar , Prosiding Semirata FMIPA Universitas Lampung, 2013, 45-51

[33]. Degroot-Hedlin, Catherine and Steven Constable, Occam's Inversion and the North American Central Plains Electrical Anomaly, Journal Geomagnetic Geoelectric, 1993, 45, 985-999.

[34]. Degroot-Hedlin, C and S. Constable, Occam's Inversion to Generate Smooth,TwoDimensional Models from Magnetotelluric data, Jurnal Geophysics, 1990, 55(12), 16131624

[35]. Putri, R.K., Akmam, dan Harman Amir, Estimasi Struktur Batuan Menggunakan Smoothness-Constraint Least-Squares
Inversion Data Geolistrik Tahanan Jenis Konfigurasi Schlumberger di Bukit Lantiak Padang Selatan, Pillar of Physics, 2014, 2, 7380

[36]. Gad El-Qady, Chika Sakamoto, and Keisuke Ushijima, 2-D Inversion of VES data in Saqqara Archaeology Area, Egypt, Earth Planets Space, 1999, 51, 1091-1098,.

[37]. Koefoed, O., Geosounding Principles, Resistivity Sounding Measurements. Amsterdam., e-Book, 1979.

[38]. Loke, M.H., Barker, R.D.,. Rapid least-squares inversion of apparent resistivity pseudosections by a Quasi-Newton method. Geophysical Prospecting, 1996, 44, 131-152. 